\title{
Índices morfofisiológicos e biofísicos da palma forrageira sob diferentes ambientes de crescimento
}

da Silva Lima Nunes, J.; Freire da Silva, T.G. @; Bastos de Souza, L.S.; do Nascimento Araújo Junior, G.e da Silva Salvador, K.R.

Unidade Acadêmica de Serra Talhada. Universidade Federal Rural de Pernambuco. Serra Talhada/PE. Brasil

PaLAVRAS CHAVE ADICIONAIS

Cobertura morta.

Irrigação.

Nopalea sp.

Opuntia sp.

Taxas de crescimento.

\section{RESUMO}

As características morfofisiológicas e biofísicas de clones de palma forrageira foram investigadas neste estudo como indicativo à adequação do manejo da cultura sob prática de irrigação e uso de cobertura morta. O experimento foi conduzido com os clones IPA Sertânia e Miúda, ambos Nopalea cochenillifera (L.) Salm-Dick, e Orelha de Elefante Mexicana, Opuntia stricta (Haw.) Haw., em blocos casualizados, com três repetições, e sob três ambientes de crescimento: $\mathrm{RH}(-)$ - regime hídrico inferior à normal climatológica; $\mathrm{RH}(+)$ - regime hídrico superior à normal climatológica; e, $\mathrm{RH}(+\mathrm{C})$ - regime hídrico superior à normal climatológica e adoção de cobertura morta. Medidas biométricas foram obtidas ao longo do tempo e a matéria seca na ocasião da colheita, a partir dos quais, calculou-se índices morfofisiológicos e biofísicos. Os ambientes de crescimento e sua interação com os clones afetaram apenas a magnitude do índice de distribuição dos cladódios de $5^{a}$ ordem $(p<0,05)$, mas foram decisivos na sazonalidade do acúmulo de matéria seca pelos clones. A maioria dos índices morfofisiológicos e biofísicos foi influenciada pelo tipo de clone $(p<0,05)$. Os índices biofísicos se mostram ótimas opções de substituição ou complementação dos índices morfofisiológicos quando se dispõem apenas de dados biométricos e de matéria seca da ocasião da colheita da palma forrageira.

\section{Morphophysiological and biophysical indices of the forage cactus under different growth environments}

\begin{abstract}
SUMMARY
The morphophysiological and biophysical characteristics of forage cactus clones were investigated in this study as indicative of the adequacy of crop management under irrigation practice and the use of mulching. The experiment was conducted with the clones IPA Sertânia and Miúda, both Nopalea cochenillifera (L.) Salm-Dick, and Orelha de Elefante Mexicana, Opuntia stricta (Haw.) Haw., in a randomized block with three replicates and under three growth environments: $\mathrm{RH}(-)$ - water regime lower than the normal climatological; $\mathrm{RH}(+)$ - water regime higher than the climatological normal; and, $\mathrm{RH}(+\mathrm{C})$ - water regime superior to the climatological normal and adoption of mulching. Biometric measurements were obtained over time and dry matter at the time of harvest. From these data morphophysiological and biophysical indices were calculated. Growth environments and their interaction with the clones only affected the magnitude of the distribution index of the cladodes of the 5 th order $(p<0.05)$, but they were decisive in the seasonality of the accumulation of dry matter by the clones. Most morphological and biophysical indices were influenced by clone type $(p<0.05)$. The biophysical indices are great options for replacing or complementing the morphophysiological indices when biometric and dry matter data are available only when the forage cactus is harvested.
\end{abstract}

\section{INTRODUÇÃO}

A palma forrageira é uma planta dotada do mecanismo ácido das crassuláceas, que a confere elevada tolerância a ambientes quentes e com déficit hídrico (Nobel; Zutta, 2008). Apesar disso, nos últimos anos, com os sucessivos eventos de seca no Semiárido brasileiro, o uso de irrigação complementar no sistema de produção da palma tem aumentado (Queiroz et al., 2015; Lima et al., 2016; Pereira et al., 2015; Rocha et al., 2017). Essa prática em associação à cobertura morta pode promover benefícios para a interface planta-ambiente (Carvalho et al., 2017), proporcionando maior desenvolvimento das plantas, bem como acúmulo de matéria seca (Amorim et al., 2017). Entretanto, ainda há lacunas sobre o efeito destas práticas na dinâmica do crescimento da palma em distintos ambientes de cultivo. Flores-Hernández et al. (2014) não encontraram efeito do incremento de lâminas de irrigação (entre $760 \mathrm{~mm}$ ano-1 e $1380 \mathrm{~mm}$ ano-1) sobre o crescimento e a produtividade de clones de palma. Resultados similares foram observados por Queiroz et al. (2015), avaliando a Opuntia stricta (Haw.) Haw. com lâminas entre $976 \mathrm{~mm}$ ano-1 e $1202 \mathrm{~mm}$ ano-1. Amorim et al. (2017) citam incrementos significativos no acúmulo de matéria seca pela palma em decorrência do uso de 
cobertura morta. No geral, a baixa disponibilidade de água pode induzir modificações morfofisiológicas nas plantas como estratégia de tolerância ao estresse (Pereira et al., 2015).

O entendimento da resposta de culturas agrícolas a mudanças de práticas de manejo deve está atrelado a análises de crescimento. Esta técnica permite avaliar a produção biológica das plantas em distintas condições ambientais (Silva et al., 2009; Silva et al., 2014a). Para isso, medidas biométricas e de matéria seca repetidas no tempo e modelos matemáticos descrevem a expansão espaço-temporal da cultura pela mensuração de taxas como a de crescimento absoluto (TCA) e relativo (TCR), taxa de assimilação líquida (TAL) e área foliar especifica (AFE), conforme descritas por Silva et al. (2009). Esses índices permitem avaliar a dinâmica de crescimento das plantas e já foram aplicados para outras culturas (Teixeira et al., 2015; Silva et al., 2014a). Para a palma, Queiroz et al. (2015) avaliaram o efeito de lâminas de irrigação no crescimento da O. stricta, porém não estudaram a sazonalidade de suas características morfológicas. Almeida (2011) aplicou índices morfofisiológicos para a O. fícus indica (L.) Mill., cv. Gigante, e N. cochenillifera (L.) Salm-Dick, cv. Miúda, sob distintos manejos de adubação orgânica e, ou, mineral, e em sequeiro. Amorim et al. (2017) sugerem o uso da taxa de acúmulo de matéria seca na definição do momento de corte da palma.

Além dos índices morfofisiológicos TCA, TCR, TAL e AFE, que são tradicionais na análise de crescimento, índices biofísicos podem ser aplicados: produtividade de assimilação liquida (PAL), índice de cobertura do solo (ICS), índice de volume de produção de matéria seca (IVP), e os índices de distribuição da área fotossintetizante da planta (IDAF) e de distribuição dos cladódios da planta (IDCP). Estes índices possuem a vantagem de requerer apenas de dados biométricos e, ou, de matéria seca da ocasião da colheita, substituindo ou complementando os índices morfofisiológicos. Segundo Queiroz et al. (2015), os índices biofísicos permitem avaliar o volume de ocupação da massa seca (IVP) e da mesma acima da superfície do solo (ICS), a sua capacidade produtiva em função da área fotossintetizante disponível (PAL), assim como a distribuição dos cladódios na planta (IDCP) e as suas contribuições no índice da área do cladódio (IDAF).

As características morfofisiológicas e biofísicas de clones de palma forrageira em resposta a alterações do ambiente de crescimento foram investigadas neste estudo como indicativo à adequação do manejo da cultura sob prática de irrigação e uso de cobertura morta.

\section{MATERIAL E MÉTODOS}

O estudo foi conduzido em uma área experimental do Instituto Agronômico de Pernambuco, no município de Serra Talhada, estado de Pernambuco, Brasil. O clima da região, de acordo com a classificação Climática de Köppen, é do tipo BSh (Alvares et al., 2014), semiárido com precipitação anual de 667,2 mm ano-1, temperatura média do ar de $25,8^{\circ} \mathrm{C}$ e umidade relativa do ar de 62,7\%. De acordo com a Embrapa (2006), o solo experimental é do tipo Argissolo Vermelho Amarelo Eutrófico franco arenoso.

A área experimental foi implantada em abril de 2010 com clones de palma forrageira (IPA-Sertânia e Miúda, ambas N. cochenillifera (L.) Salm-Dick, e a Orelha de Elefante Mexicana, O. stricta (Haw.) Haw.), no espaçamento de 1,6 x 0,2 m, enterrando 50\% do cladódio no solo. Durante os dois primeiros anos, a cultura foi conduzida em sequeiro, sendo colhida no final do março de 2012, deixando-se apenas os cladódios basais. Nessa ocasião foi dado início ao presente estudo. Nos primeiros sete meses (entre abril e outubro de 2012), a cultura foi mantida em condições de sequeiro,



Figura 1. Precipitação pluviométrica, irrigação e evapotranspiração de referência do município de Serra Talhada, Pernambuco, Brasil. (Rainfall, irrigation and reference evapotranspiration of the municipality of Serra Talhada, State of Pernambuco, Brazil).

passando, a ser irrigada a partir de novembro 2012. A nova colheita foi realizada em março de 2014, quando resultou em um ciclo de 24 meses. As condições meteorológicas e lâminas de irrigação ao longo do período experimental são demonstradas na Figura 1.

O experimento foi disposto em delineamento de bloco ao acaso, contendo três repetições, em arranjo fatorial $3 \times 3$, com parcela subdividida. As parcelas foram compostas por três ambientes de crescimento e as subparcelas pelos clones. Os ambientes de crescimento foram denominados:

- $\mathrm{RH}(-)$ : regime hídrico inferior à normal climatológica $(667,2 \mathrm{~mm}$ ano-1), onde os clones receberam ao longo do tempo o equivalente a $309 \mathrm{~mm}$ ano-1, apenas por precipitação pluviométrica;

- $\mathrm{RH}(+)$ : regime hídrico superior à normal climatológica $(667,2 \mathrm{~mm}$ ano-1), que além dos eventos de precipitação pluviométrica, os clones receberam lâminas complementares e regulares de irrigação ao longo do tempo, resultando $911 \mathrm{~mm}$ ano-1 (precipitação pluviométrica mais irrigação);

- RH(+C): A terceira condição, o regime hídrico foi superior à normal climatológica $(667,2 \mathrm{~mm}$ ano-1), os clones receberam o equivalente a $911 \mathrm{~mm}$ ano-1 (preci- 
pitação pluviométrica mais lâminas complementares e regulares de irrigação) e adotou-se o uso de cobertura.

O ensaio foi composto por nove tratamentos com subparcelas de quatro fileiras contendo 20 plantas cada fileira e totalizado 27 subparcelas. Cada subparcela apresentou $25,6 \mathrm{~m} 2(4 \times 6,4 \mathrm{~m})$ de área total e 10,24 m2 $(3,2 \times 3,2 \mathrm{~m})$ de área útil.

As irrigações foram realizadas por meio de um sistema de gotejamento, com gotejos a $0,2 \mathrm{~m}$ entre si, vazão de 1,35 L h-1 e coeficiente de uniformidade de $93 \%$, quando mantido a $100 \mathrm{kPa}$. As mangueiras gotejadoras foram dispostas adjacentes à fileira de cultivo. As lâminas de irrigação foram calculadas diariamente com base em 35\% da evapotranspiração de referência (ETo), e repostas em dias alternados. A ETo foi estimada a partir do método de Penman Monteith (Allen et al., 1998) e de dados meteorológicos coletados em uma estação automática a $1,5 \mathrm{~km}$ da área e pertencente ao Instituto Nacional de Meteorologia. A cobertura morta, por sua vez, foi imposta com a deposição de plantas espontâneas de áreas adjacentes, resultando o equivalente a 18 t ha- 1.

Práticas culturais de limpeza, controle de pragas e ervas espontâneas foram realizadas, e adubações de 50 $\mathrm{kg}$ ha-1 conduzidas com a formulação 14-0-18 (N:P:K) nos quatro primeiros meses.

Dados de biometria no início do ciclo, imediatamente após o primeiro corte da cultura, e 13 mensurações ao longo do tempo foram feitas a partir de março de 2013. Nestas medidas, registrou-se o comprimento, largura e perímetro dos cladódios de ramificações representativas de três plantas por subparcela, a altura e largura das plantas, o número total de cladódios por planta e por ordem de surgimento. Com os dados biométricos foram estimados as áreas dos cladódios e o índice de área do cladódio (IAC, m2 m-2), conforme procedimentos de Silva et al. (2014b) e Pinheiro et al. (2014).

Na ocasião da colheita, todas as plantas da área útil de cada subparcela foram pesadas, e uma amostra de cinco cladódios do terço médio das plantas foi fragmentada e acondicionada em sacos de papel, e encaminhados para estufa de ventilação forçada a $65^{\circ} \mathrm{C}$, até obtenção de massa constante. Esses procedimentos foram descritos por Silva et al. (2014b) e Silva et al. (2015). Os dados de matéria seca (W) foram expressos em $\mathrm{t}$ ha-1, assumindo uma densidade populacional de 31.250 plantas ha-1. Estes dados de matéria seca foram usados para a estimativa da matéria seca acumulada pela cultura ao longo do tempo, a partir de equações matemáticas, conforme Amorim et al. (2017).

Com os dados de biometria e de matéria seca foram calculados índices morfofisiológicos e biofísicos. Para isso, equações sigmoides em função dos dias após o corte (DAC) foram ajustadas aos dados de $\mathrm{W}$ e IAC, e, em seguida, derivadas, resultando nas taxas diárias $\mathrm{dW} / \mathrm{dt}$ e dIAC/dt. Estes dados foram integrados em intervalos mensais, totalizando 24 meses. Os índices morfofisiológicos da taxa de crescimento absoluta (TCA, t ha-1 mês-1), taxa de crescimento relativo (TCR, t t-1 mês-1), taxa de assimilação líquida (TAL, t ha-1 mês-1) e área específica do cladódio (AEC, ha t-1). A TCA foi a própria $\mathrm{dW} / \mathrm{dt}$, antes descrita. A razão entre $\mathrm{dW} / \mathrm{dt}$ e os valores absolutos de $\mathrm{W}$ resultaram na TCR. A TAL foi obtida pela razão entre a $\mathrm{dW} / \mathrm{dt}$ e os valores absolutos de IAC. A AEC foi resultante da razão entre $\mathrm{dIAC} / \mathrm{dt}$ (em ha ha-1) e dW/dt. Esses índices foram calculados ao longo do ciclo da cultura. Os índices biofísicos, por sua vez, foram calculados a partir dos dados da ocasião da colheita, conforme procedimentos citados por Queiroz et al. (2015).

O primeiro índice calculado, a produtividade de assimilação líquida (PAL, t ha-1) reflete a capacidade produtiva da cultura em função da área fotossintetizante disponível (1)

em que, W é matéria seca da planta (t ha-1); e, IAC é o índice de área do cladódio (ha ha-1 equivalente a $\mathrm{m} 2 \mathrm{~m}-2)$.

$\mathrm{O}$ índice de cobertura do solo (ICS, em \%), que concebe a ocupação da cultura acima da superfície do solo, foi obtido pela equação (2)

em que, LP é a largura da planta (m); e E é espaçamento entre as fileiras de cultivo $(\mathrm{m})$.

O índice de volume de produção (IVP, dm3 t-1) reflete o volume ocupado pela matéria seca da planta (3)

em que, 1000 é o fator de conversão de unidades (m3 para dm3); AP é a altura da planta (m); DFP é a densidade final de plantas (unidades ha-1).

$\mathrm{O}$ índice de distribuição dos cladódios (IDCP, em \%) indica a alocação dos cladódios das diferentes ordens de surgimento na planta (4)

em que, NCOn e NCT são os números de cladódios por ordem de surgimento " $\mathrm{n}$ " e total da planta, respectivamente.

A distribuição da área fotossintética (IDAF, em \%), que confere a contribuição das áreas dos cladódios por ordem de surgimento no índice da área do cladódio da planta (5)

em que, IAC-On é o índice da área do cladódio na ordem " $\mathrm{n}$ ".

Os índices morfofisiológicos e biofísicos dos clones nos distintos ambientes de crescimento foram comparados por meio da análise de variância e, em caso de significativa, foi aplicado o teste de média de Tukey, ao nível de 5\% de probabilidade, por meio do aplicativo XLSTAT.

\section{RESULTADOS}

Os ambientes de crescimento influenciaram apenas o índice de distribuição dos cladódios de $5^{\mathrm{a}}$ ordem (IDCP-O5) ( $\mathrm{p}<0,05$, Tabela I). A maioria dos índices morfofisiológicos e biofísicos foi afetada pelo tipo de clone ( $\mathrm{p}<0,05$, Tabela II): taxa de crescimento absoluto (TCA), índice de cobertura do solo (ICS) e, os índices de distribuição dos cladódios na planta (IDCP) e da área fotossintética (IDAF), a depender da ordem de surgimento. As taxas de crescimento relativo (TCR = 0,21 $\pm 0,04$ t t-1 mês-1) e de assimilação líquida (TAL 
$=1,80 \pm 0,83$ t ha-1 mês- 1 ) não foram afetadas pelos fatores analisados, mas esse último índice apresentou sazonalidade marcante, juntamente com a TCA. A produtividade de assimilação líquida $(\mathrm{PAL}=0,13 \pm 0,07 \mathrm{~kg}$ $\mathrm{m}-2)$, área específica do cladódio $(\mathrm{AEC}=0,20 \pm 0,16$ ha

Tabela I. Efeito do ambiente de crescimento $\left(\mathrm{RH}^{(-}\right.$ ): regime hídrico inferior à normal climatológica; $\mathrm{RH}^{(+)}$: regime hídrico superior à normal climatológica; $\mathrm{e}, \mathrm{RH}^{(+\mathrm{C})}$ : regime hídrico superior à normal climatológica e adoção de cobertura morta) nas características morfogênicas de clones de palma forrageira. (Effect of the growth environments (WR (-): rainfall below normal climatological; $\mathrm{WR}^{(+)}$: rainfall above normal climatological; and, $W R^{(-C)}$ : rainfall higher than normal climatological and adoption of mulching) on the morphogenic characteristics of forage cactus clones).

\begin{tabular}{lllll}
\hline Variável & Unidade & $\mathrm{RH}^{(-)}$ & $\mathrm{RH}^{(+)}$ & $\mathrm{RH}^{(+C)}$ \\
IDCP-NC5 & $\%$ & $0 \mathrm{~b}$ & $3,3 \mathrm{a}$ & $0 \mathrm{~b}$
\end{tabular}

IDCP-NC5 = índice de distribuição dos cladódios de $5^{\mathrm{a}}$ ordem. Médias seguidas pelas mesmas letras minúsculas na horizontal não diferem estatisticamente entre si pelo teste de Tukey $(P>0,05)$.

\begin{tabular}{|c|c|c|c|c|}
\hline \multicolumn{5}{|c|}{$\begin{array}{l}\text { Tabela II. Efeito de clone (IPA Sertânia: IPA; Miúda: } \\
\text { MIU e Orelha de Elefante Mexicana: OEM) sobre } \\
\text { as características morfológicas da palma forrageira } \\
\text { sob distintos ambientes de crescimento (Effect of } \\
\text { clone (IPA Sertânia': IPA; 'Miúda': MIU and 'Orelha de Elefante } \\
\text { Mexicana': OEM) on the morphological characteristics of forage } \\
\text { cactus under different growth environments). }\end{array}$} \\
\hline Variável & Unidade & IPA & MIU & OEM \\
\hline TCA & t ha ${ }^{-1}$ mês $^{-1}$ & $0,39 b$ & $0,38 b$ & $0,72 a$ \\
\hline ICS & $\%$ & $17,5 b$ & $31,5 a$ & $27,3 a b$ \\
\hline IDCP-O1 & $\%$ & $36 a$ & $17 \mathrm{~b}$ & $43 a$ \\
\hline IDCP-O2 & $\%$ & $44 a$ & $28 b$ & $42 a$ \\
\hline IDCP-O3 & $\%$ & $12 b$ & $35 a$ & $6 b$ \\
\hline IDCP-O4 & $\%$ & $0,5 b$ & $12 a$ & $0,5 b$ \\
\hline IDCP-O5 & $\%$ & $0 b$ & $3 a$ & $\mathrm{Ob}$ \\
\hline IDAF-OB (\%) & $\%$ & $10 a$ & $4 b$ & $8 a b$ \\
\hline IDAF-O1 (\%) & $\%$ & $41 a$ & $16 \mathrm{~b}$ & $45 a$ \\
\hline IDAF-O3 (\%) & $\%$ & $9 b$ & $32 a$ & $5 b$ \\
\hline IDAF-O4 (\%) & $\%$ & $0,2 b$ & $13 a$ & $0,3 b$ \\
\hline IDAF-O5 (\%) & $\%$ & $0 \mathrm{~b}$ & $3 a$ & b \\
\hline
\end{tabular}

TCA - taxa de crescimento absoluto, ICS - índice de cobertura do solo, IDCP - índice de distribuição dos cladódios na planta, IDAF - índice de distribuição da área fotossintética, OB - cladódio basal, O1 - $1^{\mathrm{a}}$ ordem, O2 - $2^{\mathrm{a}}$ ordem, O3 - $3^{\mathrm{a}}$ ordem, O4 $-4^{\mathrm{a}}$ ordem e O5 - $5^{\mathrm{a}}$ ordem. Médias seguidas pelas mesmas letras minúsculas na horizontal não diferem estatisticamente entre si pelo teste de Tukey $(P>0,05)$.
Tabela III. Características morfológicas da palma forrageira sobre efeito da interação de diferentes ambientes de crescimento $\left(\mathrm{RH}^{(-)}\right.$: regime hídrico inferior à normal climatológica; $\mathrm{RH}^{(+)}$: regime hídrico superior à normal climatológica; $\mathrm{e}, \mathrm{RH}^{(+\mathrm{C})}$ : regime hídrico superior à normal climatológica, mas com o uso de cobertura morta) e de clones (IPA Sertânia: IPA; Miúda: MIU e Orelha de Elefante Mexicana: OEM) (Morphological characteristics of the forage cactus on the effect of the interaction of different growth environments (WR ${ }^{(-)}$: rainfall below normal climatological; $\mathrm{WR}^{(+)}$: rainfall above normal climatological; and, WR ${ }^{(-C)}$ : rainfall higher than normal climatological and adoption of mulching) and clones ('IPA Sertânia': IPA; 'Miúda': MIU and 'Orelha de Elefante Mexicana': OEM)).

\begin{tabular}{lllll}
\multirow{2}{*}{ Variável } & \multicolumn{5}{c}{ Clones } \\
& $\begin{array}{l}\text { Am- } \\
\text { bien- } \\
\text { tes }\end{array}$ & & & \\
& & & & \\
& $\mathrm{RH}^{(-)}$ & $0 \mathrm{Aa}$ & $1 \mathrm{Ba}$ & $0 \mathrm{Aa}$ \\
IDCP-O5 (\%) & $\mathrm{RH}^{(+)}$ & $0 \mathrm{Ab}$ & $3 \mathrm{ABa}$ & $0 \mathrm{Ab}$ \\
& $\mathrm{RH}^{(+C)}$ & $0 \mathrm{Ab}$ & $6 \mathrm{Aa}$ & $0 \mathrm{Ab}$
\end{tabular}

IDCP-O5 = índice de distribuição dos cladódios de $5^{\text {a }}$ ordem . Médias seguidas pelas mesmas letras maiúsculas, na vertical, e minúsculas, na horizontal, não diferem estatisticamente entre si $(P>0,05)$ pelo Teste de Tukey.

$\mathrm{t}-1$ ), índice de volume de produção (IVP $=1,24 \pm 0,16$ dm3 t-1) e o índice de distribuição da área fotossintética dos cladódios de $2^{\mathrm{a}}$ ordem (IDAF-O2 = $38 \pm 13 \%$ ) também não foram afetados pelos tipos de ambiente e clone $(p>0,05)$. Houve interação do ambiente e clone apenas para o IDCP-O5 ( $p<0,05$, Tabela III).

Dentre os índices morfofisiológicos para a palma forrageira, a TCA, ou seja, a capacidade de acúmulo de matéria seca por unidade de área e tempo, embora não significativa, foi $38 \%$ e $47 \%$ maior nos ambiente de crescimento com lâminas regulares de irrigação e adoção de cobertura morta $(\mathrm{RH}(+\mathrm{C}): 0,62 \pm 0,27 \mathrm{t}$ ha- 1 mes-1) do que nos ambientes $\mathrm{RH}(+)(0,45 \pm 0,20 \mathrm{t}$ ha- 1 mes-1) e RH(-) $(0,42 \pm 0,21$ t ha- 1 mes- 1$)$. Os clones IPA Sertânia (IPA) e MIU exibiram TCA's bem similares $(0,38 \pm 0,20$ t ha-1 mes-1), mas, em média, 89\% inferior à Orelha de Elefante Mexicana (OEM) (Tabela II). A TCA, independente do ambiente e clone, foi lenta no início do ciclo, atingindo maiores magnitudes no $2^{\circ}$ ano ( $13^{\circ}$ mês ao $24^{\circ}$ mês) (Figuras 2A, 2 B e 2C). Porém, a $\mathrm{OEM}$ no $\mathrm{RH}(+\mathrm{C})$ atingiu maiores antecipação e magnitude da TCA (Figura 2C), quando comparado aos demais clones e ambientes. Para a IPA, a maximização da TCA no ambiente RH(-) ocorre em torno dos 12 meses, mas nos ambientes $\mathrm{RH}(+)$ e $\mathrm{RH}(+\mathrm{C})$, além das TCA's superiores, percebeu-se uma prolongação do período de crescimento. Na Miúda, a tendência foi inversa; no ambiente RH(-) a maior TCA ocorre no final do $2^{\circ}$ ano, enquanto, nítida antecipação foi averiguada nos ambientes $\mathrm{RH}(+)$ e $\mathrm{RH}(+\mathrm{C})$.

Independentemente do ambiente de crescimento e clone, a velocidade de acúmulo de massa seca, ou seja, a TCR obteve maiores valores no início do ciclo $(1,0 \mathrm{t}$ 
t-1 mês-1), reduzindo ao longo do tempo, mesmo com a aplicação dos tratamentos (Figuras 2D, 2E e 2F).

Os clones de palma, nos três ambientes de crescimento, exibiram maiores magnitudes de TAL no $1^{\circ}$ ano e menores no $2^{\circ}$ ano do ciclo (Figuras $3 \mathrm{~A}, 3 \mathbf{B}$ e 3C). Ainda que não significativa a diferença entre os valores da TAL dos ambientes de crescimento e clones, uma clara mudança foi verificada, onde a TAL foi ligeiramente superior no $\mathrm{RH}(-)$ e tendeu diminuir no $\mathrm{RH}(+)$ e $\mathrm{RH}(+\mathrm{C})$.

A sazonalidade da AEC não variou entre os ambientes de cultivo (Figuras 3C, 3D e 3E), mas embora não significativas, percebeu-se maiores magnitudes da AEC no início do ciclo para a OEM, especialmente no ambiente RH(-). Para os demais clones, o AEC pouco variou ao longo do tempo.

Em termos dos índices biofísicos, o ICS foi superior para o clone MIU, ainda que não tenha apresentado diferença significativa da OEM (Tabela II). Os IDCP's

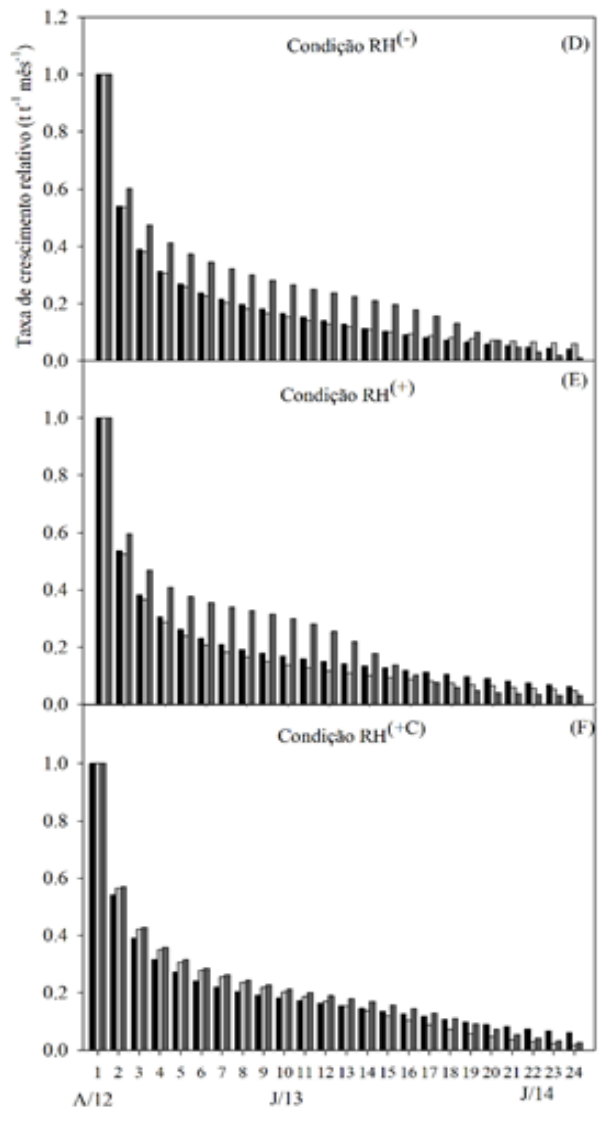

Figura 2 D, E, F. Taxas de crescimento relativo (D, E, F) de clones de palma forrageira (IPA Sertânia: IPA; Miúda: MIU e Orelha de Elefante Mexicana: OEM) sob distintos ambientes de crescimento (RH(-): regime hídrico inferior à normal climatológica; $\mathrm{RH}(+)$ : regime hídrico superior à normal climatológica; $\mathrm{e}$, $\mathrm{RH}(+\mathrm{C})$ : precipitação superior à normal climatológica e adoção de cobertura morta) (Relative (D, E, F) growth rates of forage cactus clones ('IPA Sertânia': IPA; 'Miúda': MIU and 'Orelha de Elefante Mexicana': OEM) under different growth environments (WR(-): rainfall below normal climatological; WR $(+)$ : rainfall above normal climatological; and, WR(-C): rainfall higher than normal climatological and adoption of mulching). revelaram que os clones IPA e OEM possuem maior emissão de cladódios de ordens inferiores $\left(1^{\mathrm{a}}\right.$ e $2^{\mathrm{a}}$ ordens) e a MIU de ordens superiores $\left(3^{\mathrm{a}}, 4^{\mathrm{a}}\right.$ e $5^{\mathrm{a}}$ ordens) (Tabela II), e por isso, as suas maiores contribuições na área fotossintética da planta, como averiguado pelo IDAF. O uso de irrigação sem cobertura morta, $\mathrm{RH}(+)$ aumentou o IDCP-O5 (Tabela I), especialmente por causa da maior emissão de cladódios desta ordem pelo clone Miúda (MIU) (Tabela III).

\section{DISCUSSÃO}

A falta de efeito dos ambientes de crescimento sobre índices morfofisiológicos e biofísicos da palma forrageira (exceção do IDCP-O5, Tabela I), também foi relatada por Flores-Hernández et al. (2004) e Queiroz et al. (2015) sob diferentes regimes hídricos, confirmando a alta resistência desta espécie (Snyman, 2007). As principais diferenças foram exibidas entre os clones que, na maioria das vezes, possuem adaptações peculiaridades as suas morfologias (Snyman, 2006; Falcão et al., 2013). Segundo Scalisi et al. (2016), a dinâmica de crescimento da palma depende de fatores como idade, regime hídrico e temperatura do ar. Estes autores citam que a restrição hídrica reduz a condutância estomática, conteúdo relativo de água, espessura e a taxa de crescimento dos cladódios, mas redução progressiva das flutuações do crescimento dos cladódios sob condições severas de déficit hídrico são verificadas com o seu envelhecimento ( 1 ano de idade, espessura de $8,0 \mathrm{~mm}$ e conteúdo relativo de água acima de $45 \%$ nos cladódios). Sob plena de disponibilidade de água, a dinâmica de crescimento da palma tem boa relação com a temperatura do ar. Por sua vez, Amorim et al. (2017) atestaram o efeito da cobertura morta na intensificação da emissão de cladódios de $2^{\mathrm{a}}$ ordem e redução do número de cladódios de $3^{\mathrm{a}}$ ordem, e sobre o incremento da taxa acúmulo de matéria seca dessa cultura, resultado de melhorias nas relações hídricas como discutido por Carvalho et al. (2017), e semelhante ao observado para outras culturas (Zhang et al., 2017).

A maior capacidade de acúmulo de matéria seca (TCA) pela OEM, em relação aos clones da espécie $\mathrm{N}$. cochenillifera (IPA e MIL, Tabela II) também foi constatada por Silva et al. (2015), em condições de sequeiro, e por Queiroz et al. (2015) em cultivo irrigado, justificado pela quantidade de cladódios da OEM superior a IPA e maiores dimensões quando comparada a MIU (Silva et al., 2014b; Pinheiro et al., 2014). A baixa TCA da palma no início do ciclo (Figuras 2A, 2B e 2C) é um fenômeno típico entre as comunidades vegetais, como verificado por Souza et al. (2014) e Barbosa et al. (2013). Segundo Souza et al. (2014), as plantas possuem três fases de crescimento bem definidas: inicial lenta, de crescimento rápido e estabilização, e diminuição rápida. O pequeno número de células no início induz a uma reduzida divisão celular, o que resulta em baixa área foliar e, consequentemente, menor aproveitamento da radiação e nutrientes, diminuindo a fotossíntese líquida da planta (Aumonde et al., 2013). A máxima TCA aqui obtida para palma $(2,15 \mathrm{t}$ ha- 1 mes- 1 para OEM no ambiente $\mathrm{RH}(+\mathrm{C})$ ) supera valores citados na literatura para plantas $\mathrm{C} 4$, como por exemplo, encontrado por Silva et al. (2014a), em cultivo de cana-de-açúcar irri- 
gada, com valor máximo de TCA de 1,254 t ha-1 mes-1 aos 200 DAC. Mesmo nos ambientes RH(-) e RH(+), a TCA máxima da palma atingiu 1,32 t ha-1 mes-1 e 1,24 t ha-1 mes-1, nessa ordem. Estes resultados mostram a alta capacidade de acúmulo de matéria seca dessa cultura por unidade de área, sobretudo com o uso da cobertura morta. Queiroz et al. (2015), para a O. stricta,

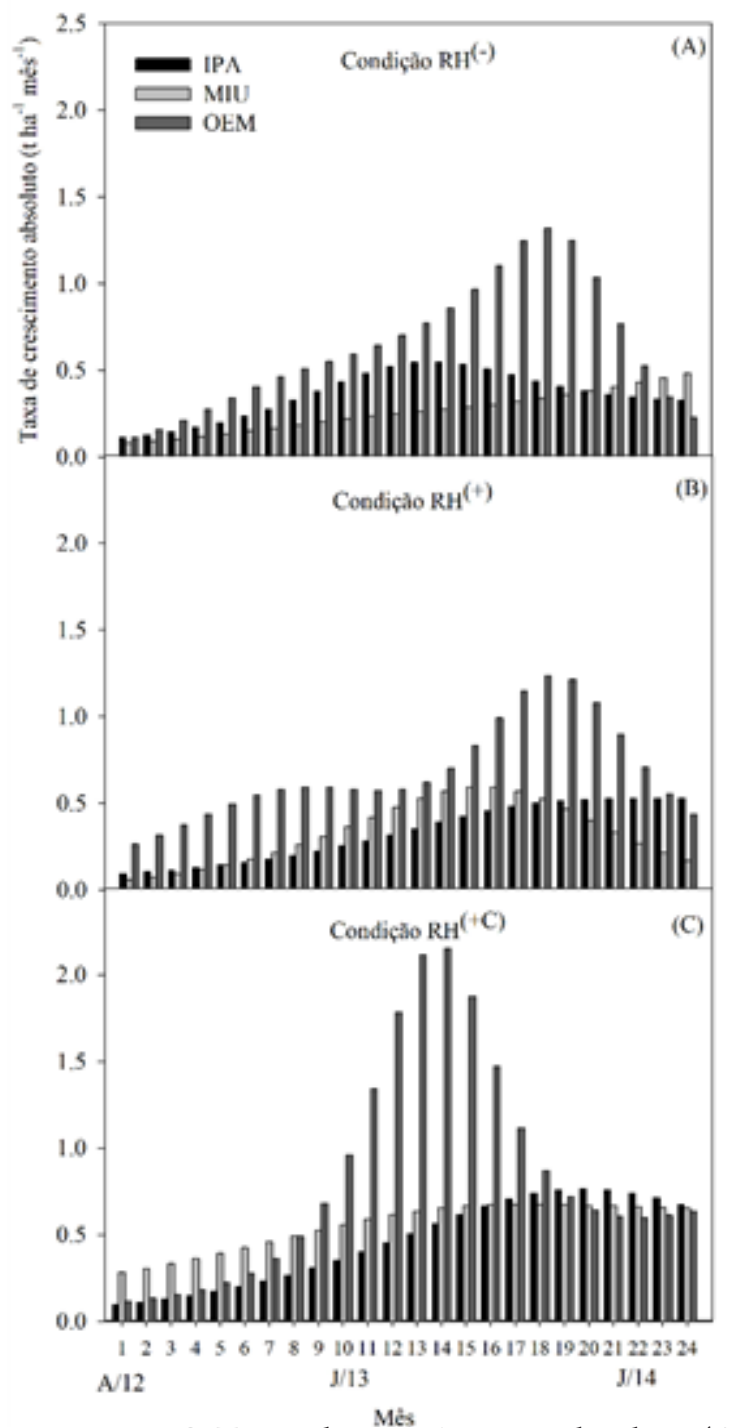

Figura 2 A, B, C. Taxas de crescimento absoluto (A, B C) de clones de palma forrageira (IPA Sertânia: IPA; Miúda: MIU e Orelha de Elefante Mexicana: OEM) sob distintos ambientes de crescimento (RH(-): regime hídrico inferior à normal climatológica; $\mathrm{RH}(+)$ : regime hídrico superior à normal climatológica; e, $\mathrm{RH}(+\mathrm{C})$ : precipitação superior à normal climatológica e adoção de cobertura morta) (Absolute $(A, B, C)$ growth rates of forage cactus clones ('IPA Sertânia': IPA; 'Miúda': MIU and 'Orelha de Elefante Mexicana': OEM) under different growth environments (WR(-): rainfall below normal climatological; WR(+): rainfall above normal climatological; and, WR(-C): rainfall higher than normal climatological and adoption of mulching). cv. OEM, no Semiárido brasileiro, durante um ciclo de 13 meses, não verificaram efeito da aplicação de lâminas regulares de $976 \mathrm{~mm}$ ano-1 e $1096 \mathrm{~mm}$ ano-1 sobre a TCA, resultando em média de 0,68 t ha- 1 mês1 , próximo ao valor obtido no presente estudo $(0,68 \mathrm{t}$ ha-1 mês-1). Almeida (2011) encontraram valores entre 0,05 e 0,25 tha-1 mês-1 para os clones 'Gigante' e MIU, durante um ciclo de 30 meses. A TCA é um ótimo indicador para definição do momento de corte da palma forrageira, como sugerido por Amorim et al. (2017).

A TCR que reflete a velocidade do incremento de matéria seca em relação ao material pré-existente, obteve maiores valores no $1^{\circ}$ ano do ciclo, variando de 0,51 t t-1 mês-1 a 0,12 t t-1 mês-1, com média de 0,32 t t-1 mês-1 (Figuras 2D, 2E e 2F). Este resultado indica a alta eficiência inicial do uso da radiação solar pelas folhas (cladódios, no caso da palma) e a sua capacidade de translocação de fotoassimilados para outros órgãos da planta (Aumonde et al., 2013; Barbosa et al., 2013). Almeida (2011), em estudo com palma forrageira, O. fícus indica, cv. 'Gigante', e N. cochenillifera, cv. Miú$\mathrm{da}$, encontrou valores de TCR em torno de $0,96 \mathrm{t} \mathrm{t}-1$ mês- 1 no $6^{\circ}$ mês após o plantio a 0,08 t t- 1 mês- 1 no $30^{\circ}$ mês, independentemente da adubação orgânica ou mineral, e do tipo de clone. A redução da TCR com a maturidade da planta é um ação comum, logo que o aparecimento de tecidos não fotossintetizantes e auto sombreamento das folhas aumentam o processo de respiração (Aumonde et al., 2013; Teixeira et al., 2015). Queiroz et al. (2015) citam valores médios de 0,55 t t-1 ha-1 para a O. stricta, cv. OEM irrigada. Snyman (2013) encontrou valores de TCR de 0,04 t t- 1 mês- 1 a $0,42 \mathrm{t}$ t-1 mês-1, a depender da espécie de palma (O. ficusindica e O. robusta) e o ano do ciclo ( $1^{\circ}$ ao $4^{\circ}$ ano), em Bloemfontein, região semiárida da África do Sul.

Para a TAL, a literatura mostra uma variação gaussiana ao longo do tempo, com momentos e máximos valores variando conforme a espécie C3 ou C4 e o manejo adotado. Porém, a maioria dos resultados aqui obtidos para a palma diverge dessa tendência, já que apresentou magnitudes mais altas no início do ciclo $(8,83 \pm 3,20$ t ha-1 mês-1) e diminuição ao longo do tempo (até $0,49 \pm 0,21$ t ha-1 mês-1) (Figuras 3A, 3B e $3 C)$. Este resultado se assemelha ao citado por Almeida (2011), com valores variando de 15 t ha-1 mês-1 a 1,5 t ha-1 mês-1, indicando tendência peculiar a esta espécie. A média aqui obtida foi de 1,80 \pm 0,83 t ha- 1 mês-1, valor este superior ao encontrado por Queiroz et al. (2015) para a O. stricta, cv. Orelha de Elefante Mexicana irrigada também no Semiárido brasileiro, de 0,67 t ha-1 mês-1 durante um ano de ciclo. A TAL representa a fotossíntese líquida, resultado do balanço entre os fotoassimilados produzidos pela fotossíntese e aqueles perdidos na respiração. Flores-Hernández et al. (2004), avaliando quatro clones da espécie O. fícus indica não averiguaram efeito de lâminas regulares e crescentes de irrigação (740 mm ano-1 a $1380 \mathrm{~mm}$ ano-1) na TAL, mas houve diferença entre os clones com valores médios do ciclo variando entre 4,29 e 7,29 t ha-1 mês-1. Esse resultado é diferente do obtido no presente estudo, logo que não foram constatadas diferenças nas eficiências fotossintéticas entre os clones. Aqui, a TAL foi calculada a partir da matéria seca 


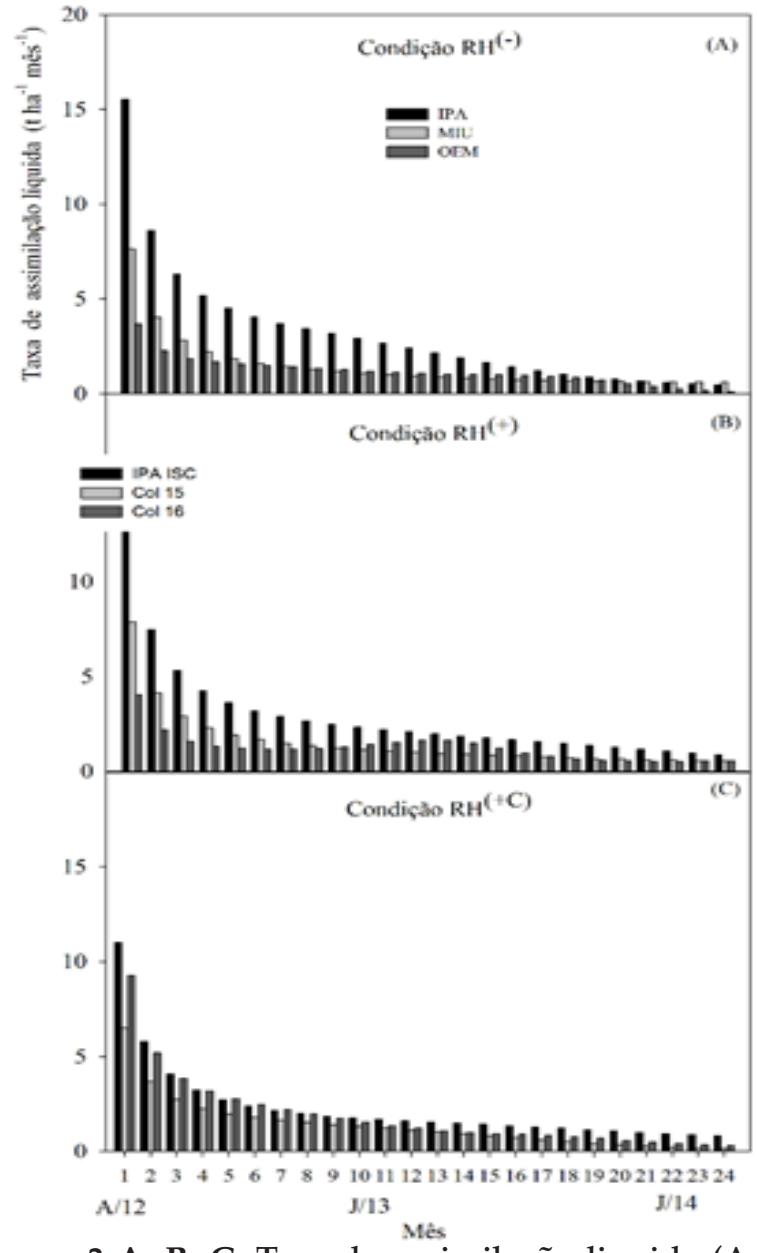

Figura 3 A, B, C. Taxa de assimilação liquida (A, B, C) de clones de palma forrageira (IPA Sertânia: IPA; Miúda: MIU e Orelha de Elefante Mexicana: OEM), sob distintos ambientes de crescimento (RH(-): regime hídrico inferior à normal climatológica; $\mathrm{RH}(+)$ : regime hídrico superior à normal climatológica; $\mathrm{e}$ $\mathrm{RH}(+\mathrm{C})$ : precipitação superior à normal climatológica e adoção de cobertura morta) (Net assimilation rate $A$, B, C) of forage cactus clones ('IPA Sertânia': IPA; 'Miúda': MIU and 'Orelha de Elefante Mexicana': OEM) under different growth environments (WR(-): rainfall below normal climatological; WR(+): rainfall above normal climatological; and, WR(-C): rainfall higher than normal climatological and adoption of mulching).

da parte área, indicando que essa similaridade esteja associada à partição de fotoassimilados para as raízes, característica essa peculiar a cada clone. A OEM que possui maior TCA também deve ser o clone que mais investe em fotoassimilados para as raízes, enquanto os clones da espécie N. cochenillifera, que possuem menores TCA's, devem apresentar menor crescimento do sistema radicular.

Essa afirmativa foi citada por Snyman (2006), que averiguaram produtividade de matéria seca de raízes e da parte aérea de $239,06 \mathrm{~kg}$ ha-1 e $2.462 \mathrm{~kg}$ ha-1 para a O. ficus-indica e de $316,62 \mathrm{~kg}$ ha-1 e $2.000 \mathrm{~kg}$ ha-1 para a O. robusta, sendo a partição de $20 \%$ e $16 \%$, respectivamente. A AEC, na maioria dos resultados, igualmente a $\mathrm{TAL}$, apresentou sazonalidade distinta à observada na literatura para espécies C3 e C4 (Silva et al., 2009; Silva et al., 2014a). A AEC reflete a dinâmica da espes-

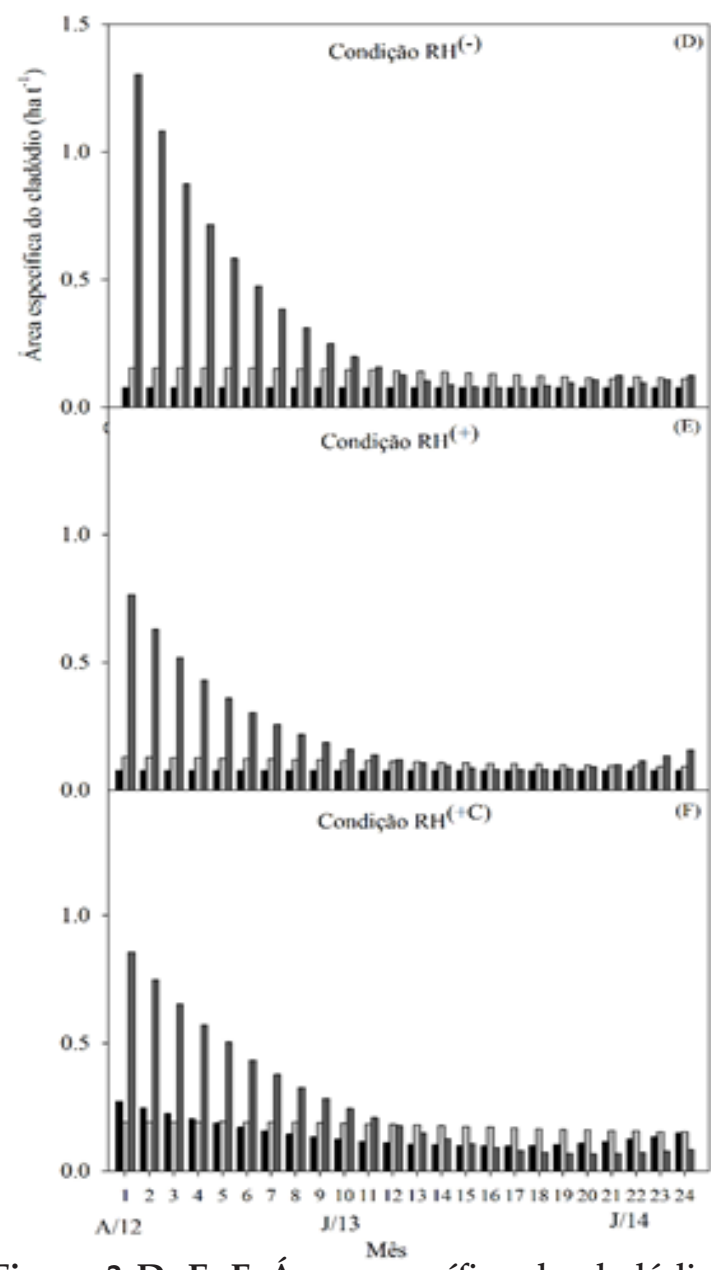

Figura 3 D, E, F. Área específica do cladódio (D, E, F) de clones de palma forrageira (IPA Sertânia: IPA; Miúda: MIU e Orelha de Elefante Mexicana: OEM), sob distintos ambientes de crescimento (RH(-): regime hídrico inferior à normal climatológica; $\mathrm{RH}(+)$ : regime hídrico superior à normal climatológica; $\mathrm{e}$, $\mathrm{RH}(+\mathrm{C})$ : precipitação superior à normal climatológica e adocão de cobertura morta) (Specific cladode area (D, E,F) of forage cactus clones ('IPA Sertânia': IPA; 'Miúda': MIU and 'Orelha de Elefante Mexicana': OEM) under different growth environments (WR(-): rainfall below normal climatological; WR(+): rainfall above normal climatological; and, WR(-C): rainfall higher than normal climatological and adoption of mulching).

sura do cladódio, sendo mais fina quanto maior a AEC e mais espessa quanto menor os seus valores. Para os clones IPA e MIU, a AEC pouco variou ao longo do tempo, ao passo que para a OEM seus valores foram maiores no início 0,97 ha t- 1 e menores ao final do ciclo $(0,12$ ha t-1) (Figuras 3D, 3E e 3F). Segundo Silva et al. (2009), este índice tende a diminuir ao longo do tempo, mesmo com a estabilização da área foliar, uma vez que há aumento dos tecidos condutores da planta e, por conseguinte, persistência do acúmulo de matéria seca. Almeida (2011) encontrou valores variando de 0,07 ha $\mathrm{t}-1$, no início, a 0,03 ha $\mathrm{t}-1$, ao final do ciclo.

Em relação aos índices biofísicos, a PAL não apresentou diferenças entre os ambientes de crescimento e clones, com média de 0,13 $\pm 0,07$ tha- 1 , indicando que a palma forrageira produz $0,13 \mathrm{t}$ de matéria seca por unidade hectare de área fotossintetizante. Este índice 
pode ser associado com a TAL, o que reflete a fotossíntese liquida da cultura e, logo, a sua eficiência da utilização da radiação.

O ICS possui relação com o IAC da cultura. Como mostram os resultados na Tabela I, os clones MIU e OEM foram aqueles com maiores magnitudes do ICS $(31,5 \%$ e $27,3 \%$, nessa ordem). O presente estudo (dados não mostrados) e outros vários trabalhos revelam a superioridade do IAC desses clones em relação à IPA (Silva et al., 2014b; Pinheiro et al., 2014; Silva et al., 2015).

O IVP é um índice que reflete o volume ocupado pela matéria seca da planta, oferecendo a ideia de densidade produtiva. Quanto menor o IVP, maior é o adensamento da produção da cultura. Este índice sofre bastante o efeito do hábito de crescimento da cultura, uma vez que este influencia no volume ocupado pela planta. A média de 1,24 $\pm 0,16 \mathrm{dm} 3 \mathrm{t}-1$, obtido no presente estudo, indica que 1,0 tonelada de massa seca produzida pela palma ocupou 1,24 dm3.

Em termos do IDCP, este índice é especifico para a palma, representando a distribuição dos cladódios ao longo da estrutura da planta. Os cladódios de $1^{\mathrm{a}} \mathrm{e}$ $2^{\mathrm{a}}$ ordens, independente do ambiente de crescimento, foram mais predominantes nos clones IPA e OEM (Tabela II). Este resultado é similar ao obtido por Queiroz et al. (2015), avaliando a OEM, mesmo sob distintas lâminas de irrigação, mostrando que esta característica é peculiar ao clone. A MIU apresentou mais emissão de cladódios de ordens superiores ( $>3^{\mathrm{a}}$ ordem). Silva et al. (2015) citam que, em sequeiro e durante 24 meses, a MIU exibiu maior emissão de cladódios em relação a IPA e OEM, desde a $2^{a}$ ordem. Aqui o IDCP-O5 da MIU sofreu efeito do ambiente $\mathrm{RH}(+)$ (Tabelas I e III), o que pode está associado ao fato da dinâmica de crescimento de cladódios mais novos responderem mais rápida a variações de regime hídrico quando comparada a cladódios mais velhos (Scalisi et al., 2016).

O IDAF completa o IDCP, logo que a contribuição da área foliar por ordem de surgimento dos cladódios depende do seu número e dimensões. As maiores contribuições para o índice da área do cladódio dos clones IPA e OEM foram dos cladódios de ordens inferiores (< $2^{\mathrm{a}}$ ordem), enquanto na MIU foram os cladódios de ordens superiores ( $>=3^{\circ}$ ordem) (Tabela II). Para a OEM, Queiroz et al. (2015) relatam resultados semelhantes. Mas, essa tendência de similaridade das ordens entre o IDCP e IDAF nem sempre pode ocorrer em função das diferenças morfológicas dos cladódios e das plantas dentre a diversidade de genótipos de palma. Algumas distinções foram identificadas por Pinheiro et al. (2014) e Silva et al. (2014b).

\section{CONCLUSÕES}

O presente estudo revelou que os índices morfofisiológicos e biofísicos da palma forrageira são mais inerentes ao tipo de clone e pouco influenciados pelo ambiente de crescimento, mas este afeta de maneira expressiva a sazonalidade do acúmulo de matéria seca. Os índices biofísicos se mostram ótimas opções de substituição ou complementação dos índices mor- fofisiológicos quando se dispõem apenas de dados biométricos e de matéria seca da ocasião da colheita da palma forrageira.

\section{AGRADECIMENTOS}

Ao Conselho Nacional de Desenvolvimento Cientifico e tecnológico (CNPq) pelo auxílio financeiro (processo $\mathrm{n}^{\circ}$. 475279/2010-7).

\section{BIBLIOGRAFIA}

Allen RG, Pereira LS, Raes D \& Smith M 1998, Crop evapotranspiration: Guidelines for computing crop water requirements, FAO, Rome, IT.

Almeida, J 201 1, A palma forrageira na região semiárida do estado da Bahia: diagnóstico, crescimento e produtividade, Tese de Doutorado, Universidade Federal do Recôncavo da Bahia, Cruz das Almas, BA, Brasil.

Alvares CA, Stape JL, Sentelhas PC, Gonçalves JLM \& Sparovek G 2014 , 'Köppen's climate classification map for Brazil', Meteorologische Zeitschrift, vol. 22, pp. 711-728.

https://dx.doi.org/10.1127/0941-2948/2013/0507

Amorim DM, Silva TGF, Pereira PC, Souza LSB \& Minuzzi RB 2017, 'Phenophases and cutting time of forage cactus under irrigation and cropping systems', Pesquisa Agropecuária Tropical, vol. 47, pp. 62-71. http://dx.doi.org/10.1590/1983-40632016v4742746

Aumonde TZ, Pedó T, Martinazzo EG, Moraes DM, Villela FA \& Lopes NF 2013, 'Análise de crescimento e partição de assimilados em plantas de maria-pretinha submetidas a níveis de sombreamento', Planta daninha, vol. 31, pp. 99-108.

http://dx.doi.org/10.1590/S0100-8358201300010001 1

Barbosa ML, Silva TGF, Silva AC, Almeida MG, Lima ALA \& Souza CAA 2013, 'Crescimento Inicial de Espécies Ocorrentes no Semiárido Brasileiro: Biomassa, Biometria e Análise Morfogênica', Revista Brasileira de Geografia Física, vol. 6, pp. 522-539.

http://www.revista.ufpe.br/rbgfe/index.php/revista/article/viewArticle/704

Benincasa MMP 2003 Análise de crescimento de plantas (noções básicas), 2nd edn, FUNEP, Jaboticabal, SP.

Carvalho AA, Silva TGF, Souza LSB, Moura MSB, Araújo GGL, Toledo MPS 2017, 'Soil moisture in forage cactus plantations with improvement practices for their resilience', Revista Brasileira de Engenharia Agricola e Ambiental, vol. 21, pp. 481-487.

http://dx.doi.org/10.1590/1807-1929/agriambi.v21n7p481-487

Falcão HM, Oliveira MT, Mergulão A, Silva MV, Santos MG 2013, 'Ecophysiological performance of three Opuntia ficus-indica cultivars exposed to carmine cochineal under field conditions', Scientia Horticulturae, vol. 150, pp. 419-424. https://doi.org/10.1016/j. scienta.2012.11.021

Flores-Hernández A, Orona-Castillo I, Murillo-Amador B, GarcíaHernández JL \& Troyo-Dieguez E 2004, 'Yield and physiological traits of prickly pear cactus 'nopal' (Opuntia spp.) cultivars under drip irrigation', Agricultural Water Management, vol. 70, pp. 97-107.

http://dx.doi.org/10.1016/j.agwat.2004.06.002

Lima GFC, Rego MMT, Dantas FDG, Lôbo RNB, Silva JGM \& Aguiar EMA 2016, 'Morphological characteristics and forage productivity of irrigated cactus pear under different cutting intensities', Revista Caatinga, vol .29, pp. 481-488. http://dx.doi.org/10.1590/1983$21252016 v 29$ n226rc

Nobel PS \& Zutta BR 2008, 'Temperature tolerances for stems and roots of two cultivated cacti, Nopalea cochenillifera and Opuntia robusta: Acclimation, light, and drought', Journal of Arid Environments, vol. 72, pp. 633-642. http://dx.doi.org/10.1016/i.jaridenv.2007.08.005 Pereira PC, Silva TGF, Zolnier S, Morais JEF \& Santos DC 2015, 'Morfogênese da palma forrageira irrigada por gotejamento', Revista Caatinga, vol. 28, pp. 184-195.

http://dx.doi.org/10.1590/1983-21252015v28n321 rc 
Pinheiro KM, Silva TGF, Sousa Carvalho HF, Santos JEO, Morais JEF, Zolnier S \& Santos DC 2014, 'Correlações do índice de área do cladódio com características morfogênicas e produtivas da palma forrageira', Pesquisa Agropecuária Brasileira, vol. 49, pp. 939-947. http://dx.doi.org/10.1590/S0100-204X2014001200004

Queiroz MG, Silva TGF, Zolnier S, Silva SMS, Lima LR \& Alves JO 2015, 'Características morfofisiológicas e produtividade da palma forrageira em diferentes lâminas de irrigação', Revista Brasileira Engenharia Agrícola e Ambiental, vol. 19, pp. 931-938.

http://dx.doi.org/10.1590/1807-1929/agriambi.v19n10p931-938.

Rocha RS, Voltolini TV \& Gava CAT 2017, 'Características produtivas e estruturais de genótipos de palma forrageira irrigada em diferentes intervalos de corte', Archivos de Zootecnia, vol. 66, pp. 363-371. http://www.uco.es/ucopress/az/index.php/az/article/view/2512

Scalisi A, Morandi B, Inglese P \& Bianco RL 2016, 'Cladode growth dynamics in Opuntia ficus-indica under drought', Environmental and Experimental Botany, vol. 122, pp. 158-167.

https://doi.org/10.1016/j.envexpbot.2015.10.003

Silva TGF, Zolnier S, Grossi JAS, Barbosa JG, Moura CRW \& Muniz MA 2009, 'Crescimento do girassol ornamental cultivado em ambiente protegido sob diferentes níveis de condutividade elétrica de fertirrigação', Revista Ceres, vol. 56, pp. 602-610.

http://www.ceres.ufv.br/ojs/index.php/ceres/article/view/3473

Silva TGF, Primo JTA, Morais JEF, Diniz WJS, Souza CAA \& Silva MC 2015, 'Crescimento e produtividade de clones de palma forrageira no semiárido e relações com variáveis meteorológicas', Revista Caatinga, Vol. 28, pp. 10-18.

https://periodicos.ufersa.edu.br/index.php/caatinga/article/ view $/ 3630$

Silva TGF, Moura MSB, Zolnier S, Souza LSB \& Carmo JFA 2014a, 'Índices morfofisiológicos e uso de radiação solar por um cultivo de cana-de-açúcar irrigada no Semiárido brasileiro', Revista Brasileira de Geografia Física, vol. 7, pp. 764-773.
http://www.revista.ufpe.br/rbgfe/index.php/revista/article/viewArticle/895

Silva TGF, Miranda KR, Santos DC, Queiroz MG, Silva MC, Neto JFC \& Araújo JEM 2014b, 'Área do cladódio de clones de palma forrageira: modelagem, análise e aplicabilidade', Brazilian Journal of Agricultural Sciences, vol. 9, pp. 633-641. http://dx.doi.org/10.5039/ agraria.v9i4a455

Snyman HA 2006, 'Root distribution with changes in distance and depth of two-year-old cactus pears Opuntia ficus-indica and O. robusta plants', South African Journal of Botany, vol. 72, pp. 434-441. https://doi. org/10.1016/i.sajb.2005.12.008

Snyman HA 2007, 'Root studies on cactus pears Opuntia ficus-indica and O. robusta along a soil-water gradiente', Haseltonia, vol. 13, pp. 64-75. https://doi.org/10.2985/1070-0048(2007)13[64:RSOCPO]2.0.CO;2 Snyman HA 2013, 'Growth rate and water-use efficiency of cactus pears Opuntia ficus-indica and O. robusta', Arid Land Research and Management, vol. 27, pp. 337-348.

https://doi.org/10.1080/15324982.2013.771232

Souza LSB, Moura MSB, Sediyama GC \& Silva TGF 2014, 'Crescimento e produtividade do milho e feijão-caupi em diferentes sistemas e disponibilidade hídrica no Semiárido', Revista Brasileira de Geografia Física, vol. 7, pp. 524-539.

Teixeira GCS, Stone LF \& Heinemann AB 2015, 'Eficiência do uso da radiação solar e índices morfofisiológicos em cultivares de feijoeiro', Pesquisa Agropecuária e Tropical, vol. 45, pp. 9-17.

http://dx.doi.org/10.1590/1983-40632015v4528297

Zhang G, Liu C, Xiao C, Xie R, Ming B, Hou P, Liu G, Xu W, Shen D, Wang K \& Li S 2017, 'Optimizing water use efficiency and economic return of super high yield spring maize under drip irrigation and plastic mulching in arid areas of China', Field Crops Research, vol. 211, pp. 137-146.

https://doi.org/10.1016/j.fcr.2017.05.026 